\title{
The Analgesic Efficacy of Ultrasound-guided Bilateral Transversus Thoracic MusclePlane Block after Open-Heart Surgeries: A Randomized Controlled Study
}

Mohamed Ahmed Hamed ( $\square$ mah07@fayoum.edu.eg )

Fayoum University Faculty of Medicine https://orcid.org/0000-0003-2951-5940

Maged Labib Boules

Fayoum University Faculty of Medicine

Mina Mahrous Sobhy

Fayoum University Faculty of Medicine

Mahdy Ahmed Abdelhady

Fayoum University Faculty of Medicine

\section{Research}

Keywords: Transversus Thoracic Muscle Plane Block, fentanyl consumption, sternotomy, postoperative pain

Posted Date: October 11th, 2021

DOI: https://doi.org/10.21203/rs.3.rs-955344/v1

License: (1) (i) This work is licensed under a Creative Commons Attribution 4.0 International License.

Read Full License 


\section{Abstract}

Background: We aimed to evaluate the analgesic efficacy of ultrasound-guided bilateral Transversus Thoracic Muscle Plane Block after Open-Heart Surgeries.

Methods: 70 patients aged above 18 years and scheduled for valve replacement or adult congenital via median sternotomy were enrolled in this study. Patients were divided into two groups, randomized by computer-generated random numbers: the block group, which had the ultrasound-guided bilateral transversus thoracic muscle plane block, and the control group, which had a sham block. The primary outcome was total fentanyl consumption in the first 24-hours. The secondary outcomes were pain score, time to the first analgesic request, time to extubation , ICU stays, and hospital stay.

Results: The total fentanyl consumption in the first 24 hours was significantly lower in the block group, with a mean difference of $-158.286(95 \% \mathrm{Cl}=(-179.271$ to $-137.300 ; p=<0.0001)$. The time to the first analgesic request was statistically significantly shorter in the non-block group (median 3 hours) than the block group (median 14 hours). During the postoperative period (0.5-24 hours), at-rest pain scores were 1.86 units lower in the block group (the estimate was $-1.80,95 \% \mathrm{Cl}=-2.14$ to $-1.45, \mathrm{t}=-10.323$ with $\mathrm{p}$ $<0.0001$ ). Likewise, pain scores with cough were 3.29 units lower in the block group (the estimate was $-3.29,95 \% \mathrm{Cl}=-3.80$ to $-2.77, \mathrm{t}=-12.703, \mathrm{p}<0.0001)$.

Conclusion: Bilateral Transversus Thoracic Muscle Plane Block is a promising and effective technique in reducing opioid consumption and controlling post-sternotomy pain after open-heart surgery via median sternotomy.

\section{Trial Registration:}

This study is registered on ClinicalTrials.gov (NCT04116554; principal investigator: Mohamed Ahmed Hamed; date of registration: October 4, 2019).

\section{Introduction}

Pain following cardiac surgery is influenced by many factors, including preoperative restlessness and anxiety, inflammatory reactions accompanied, direct tissue damage, the person's nociception threshold, and perioperative analgesia management. ${ }^{(1)}$

Intolerable postoperative pain is debilitating and may lead to many adverse outcomes, including; respiratory problems, reduced mobilization, anxiety, impaired immune response, mood instability, postoperative delirium, and an increased mortality rate. ${ }^{(2)}$

Postoperative pain management was dependent mainly on opioids, but the optimal results couldn't be achieved due to opioid-related side effects. ${ }^{(3)}$ 
The multimodal analgesic technique has emerged to avoid these adverse effects of opioids and achieve appropriate analgesia; one of its components is fascial plane blocks. ${ }^{(4)}$ Various techniques have been investigated for cardiac surgery via median sternotomy. including; paravertebral nerve block, the pecto intercostal fascial (PIF), serratus anterior, PECSI/II, erector spinae plane, and Transversus thoracic muscle plane (TTMP) block. ${ }^{(4)}$

TTMP block was first described by Ueshima et al. ${ }^{(5)}$; it can block multiple anterior branches of the intercostal nerves $\left(\right.$ T 2-6) ${ }^{(6)}$, which dominate the internal mammary region, including the sternum ${ }^{(7)}$.

We hypothesized that bilateral TTMP block would provide more effective and prolonged pain relief after sternotomy involved open-heart surgery and could be used as a part of multimodal opioid-sparing analgesia technique. Therefore, this study aimed to evaluate the analgesic efficacy of bilateral TTMP block after acute poststernotomy pain following cardiac surgery. The primary study outcome was the 24hours total fentanyl consumption.

\section{Methods}

This prospective, randomized, observer-blind study was conducted following the tenets of the Declaration of Helsinki. The study design was approved by the ethical review board of Fayoum University Hospital (D 199), and written informed consent was acquired from all patients. The study was conducted after registration on ClinicalTrials.gov (NCT04116554; principal investigator: Mohamed Ahmed Hamed; date of registration: October 4 , 2019). In this randomized prospective double-blinded study, seventy patients were scheduled for open-heart surgery, including valve replacement or adult congenital (ASD or VSD), in Fayoum university hospital from December 2019 to February 2021. This study adheres to the applicable CONSORT guidelines.

\section{Study_population:}

Seventy patients aged above 18 years and scheduled for elective cardiac surgery for valve replacement or adult congenital (VSD or ASD) via median sternotomy were included.

The exclusion criteria were as follows; emergency surgeries, re-do surgery, coagulopathy, preoperative poor left ventricular function (Ejection fraction (E.F.) < 35\%), systemic infections or infections at the site of injection, neuromuscular disease, psychiatric illnesses, narcotic dependency, allergy to the drug used and prolonged intensive care unit (ICU) stay for reasons as; heart failure and reoperation for hemostasis.

Patients were randomly divided into two groups with 1:1 allocation (block-group and non-block group) via computer-generated random numbers that were placed in separate opaque envelopes and opened by study investigators just before the block. All blocks were accomplished with the same anesthesiologist. Neither patients nor the functional data collector was aware of randomization.

\section{Anesthetic technique:}


All patients were preoperatively examined and investigated by complete blood count, coagulation profile, renal functions, and electrolytes. Electrocardiography, chest x-ray, and echocardiography were routinely done. Coronary angiography and carotid arterial duplex could be requested on-demand.

Patients were premedicated by intramuscular injection of $10 \mathrm{mg}$ morphine on the morning of the operation. Before induction of anesthesia, a five-lead electrocardiography system was applied to monitor heart rate, rhythm, and S.T. segments (leads $\mathrm{II}$ and $\mathrm{V}_{5}$ ). A pulse oximeter probe was attached, and a peripheral venous cannula was placed. A $20 \mathrm{G}$ cannula was inserted into either right or left radial artery under local anesthesia to measure arterial pressure and blood sampling. After pre-oxygenation, general anesthesia was induced by midazolam $2 \mathrm{mg}$, fentanyl $\left(10 \mu \mathrm{g} \cdot \mathrm{kg}^{-1}\right)$, propofol $\left(3 \mathrm{mg} \cdot \mathrm{Kg}^{-1}\right)$, followed by atracurium $\left(0.5 \mathrm{mg} \cdot \mathrm{kg}^{-1}\right)$.

The trachea was intubated; patients were mechanically ventilated with oxygen in the air, ventilation parameters were adjusted to achieve normocarbia. An esophageal temperature probe and a urinary catheter also had been placed.

For drug infusion, a triple-lumen central venous catheter was inserted via the right internal jugular vein.

Anesthesia was maintained by inhaled Isoflurane 0.4 to $1 \%$ and atracurium infusion of $0.5 \mathrm{mg} \cdot \mathrm{kg}^{-1} \cdot \mathrm{h}^{-1}$ for continued muscle relaxation. During extracorporeal circulation, patients have been received propofol infusion at a rate of $50-100 \mu \cdot \mathrm{kg}^{-1} \cdot \mathrm{min}^{-1}$. In addition to atracurium infusion.

Before initiation of CPB, the patients were received intravenous heparin (300-500 units. $\mathrm{kg}^{-1}$ body weight) to achieve an ACT > $480 \mathrm{~s}$. CPB was instituted via an ascending aortic cannula and a two-stage right atrial cannula. Before, during (pump blood flow: $2.41 / \mathrm{min} / \mathrm{m}^{2}$ ), and after CPB, mean arterial pressure was adjusted to exceed $60 \mathrm{mmHg}$. Cardiac arrest had been achieved with cold ante-grade blood cardioplegia. Lactate-enriched Ringer's solution was added to the CPB circuit to maintain reservoir volume when needed, and packed red blood cells would be added when hemoglobin concentration decrease to less than $7 \mathrm{~g} \cdot \mathrm{dl}^{-1}$.

After the patient rewarming to $37^{\circ} \mathrm{C}$, separation from $\mathrm{CPB}$, reversal of heparin by protamine sulfate $(1: 1)$, and sternal closure had been achieved.

The anesthesiology intern prepared the study solution, bupivacaine $0.25 \%$ or normal saline, in the operating room. For carrying out the block bilaterally, the skin on either side of the sternum was prepared with povidone-iodine solution. Then a linear ultrasound probe (Philips clear vue350, Philips Healthcare, Andover MA01810, USA.) was placed on the right and left sides at $3 \mathrm{~cm}$ from the mid sternum. The subcutaneous tissue was identified; the intermediate plane: the pectoralis major muscle, the intercostal muscles, the ribs; the deep plane, the transverse thoracic muscle, the pleura, and the lung.

Block-group: had received bilateral TTMP block. After identifying the anatomical plane between the internal intercostal and the transversus thoracic muscles, a 22-gauge short bevel needle (Spinocan, B. 
Braun Melsungen AG, Germany) was inserted between the fourth and fifth ribs connecting at the sternum.

Correct needle placement was confirmed by visualizing the needle in the plane along its entire length, and the tip of the needle between both muscles, then $1 \mathrm{ml}$ of anesthetic liquid was introduced after negative aspiration. The TTMP block was completed by injection of $20 \mathrm{~mL}$ of $0.25 \%$ bupivacaine, and the same procedure was repeated on the other side. A Local anesthetic indicated the appropriate injection spread deep to the costal cartilages and downward displacement of the pleura.

Non-block group: patients received sham block bilaterally with $20 \mathrm{ml}$ of $0.9 \%$ saline had been injected on each side.

All patients were transported to ICU for postoperative management and care. Tracheal extubation was performed when the patient met the following criteria: awake/arousable, hemodynamically stable, no active bleeding, warm peripheries, and satisfactory arterial blood gas with an $\mathrm{FIO} 2<0.5$, pressure support on ventilator reduced to $10 \mathrm{Cm} \mathrm{H}_{2} \mathrm{O}$, Positive End Expiratory Pressure 5-7 $\mathrm{CmH}_{2} \mathrm{O}$, no electrolyte abnormalities, minimal inotropic support, or no escalation in inotropic support.

Postoperative analgesia in the ICU was carried out for both groups. All patients received IV fentanyl via patient-controlled analgesia (PCA) with (conc. $10 \mu \mathrm{g} \cdot \mathrm{ml}^{-1}$, with bolus of $15 \mu \mathrm{g}$, and lockout interval 10 minutes, maximum cumulative dose of $90 \mu . \mathrm{hr}^{-1}$ and no background dose). Before extubation, analgesia was given as nurse-controlled analgesia (NCA) with the same regimen, depending on the sudden rise in H.R. or MABP $\geq 20 \%$ of the baseline. Paracetamol $1 \mathrm{gm}$ was given every 8 hours for all patients. The total $24 \mathrm{~h}$. opioid consumption was recorded.

\section{Measured parameters:}

The primary outcome was total fentanyl consumption (time frame: from ICU admission up to 24 hours). Secondary outcomes included: VAS score for sternal pain both during rest and with cough (ranging from 0 indicating no pain to 10 indicating extreme pain) measured at time intervals: 30 minutes, 1hour., 3hours., 6hours., 12 hours., and $24 \mathrm{~h}$ after extubation, time to extubation, The first analgesic request time, ICU length of stay and Total length stay of the hospital.

\section{STATISTICAL ANALYSIS:}

Before the study, the sample size was calculated. The primary outcome was the total fentanyl consumption during the first 24 hours after surgery. There were no previous studies when designing the study protocol. Therefore, we performed an external pilot study that included 9 patients in each group, with its results not included in the full-scale study. From this pilot, the total 24 hours postoperative fentanyl consumption $(\mu \mathrm{g})$ was $(371.7 \pm 150.03$ in the control group versus $260 \pm 110.45$ in the block group with a mean reduction of $30 \%$ ). The minimal sample size of patients was 31 in each group needed to get power level 0.90 and alpha level 0.05 . the calculated sample size was increased by $10 \%$ to reach 35 in each group to allow for dropouts. 
The collected data were organized, tabulated, and statistically analyzed using SPSS software statistical computer version 22 (SPSS Inc, USA). We used a two-sample t-test to compare the two groups' mean values (age, BMI, fentanyl consumption) and a Mann-Whitney $U$ test to compare medians for skewed endpoints ( time of extubation, ICU stay, and hospital stay). The chi-squared test was used to determine significance, and Qualitative data were presented as numbers and percentages (Sex, HTN, D.M., and the operation type). Linear mixed models were used to account for repeated measures of pain scores. A fixedeffect model was used for the group and a random effect model for the subject. The first analgesic request time was assessed using the Kaplan-Meier estimator; Median time and $(95 \% \mathrm{Cl})$ were estimated, and the log-rank test was performed to compare study groups. A two-sided P-value of $<0.05$ was considered statistically significant.

\section{Results}

For this study, 76 patients were assessed for eligibility based on the inclusion and exclusion criteria. Six patients were excluded; two cases with E.F. below $35 \%$, two patients declined to participate, and two due to stuck valves. The remaining 70 patients were randomly assigned into the block group, which received the block, and the Non-block group, which control group. The patients were followed up for analgesic consumption, pain scores, and ICU and hospital stay lengths. (Figure 1).

There was no statistically significant difference between the two groups regarding the demographic characteristics and operative data (Table 1). In addition, there was no statistically significant difference between the two groups regarding the time elapsed in ICU or the hospital (Table 1).

Mean (SD) total fentanyl consumption in the first 24 hours was significantly lower in the block group than the no block group $(205.7$ (73.5) $\mu \mathrm{g}$ vs. 390.9 (80.3) $\mu \mathrm{g}$, with a mean difference of $-185.143,95 \% \mathrm{Cl}=$ -221.871 to $-148.415 ; p<0.0001$ (Table 2 ).

The median estimate time to the first analgesic request was longer in the block group (14 hours, 95\% $\mathrm{Cl}$ $=12.17-15.84$ ) than in the no block group ( 3 hours, $95 \% \mathrm{Cl}=1.72-4.28$ ), $\mathrm{p}<0.0001$ (Figure 2).

Using mixed effect model during the post-operative period (0.5-24 hours), at-rest pain scores were 1.86 units lower in the Block group than Non-block group (the estimate was $-1.80,95 \% \mathrm{Cl}=-2.14$ to $-1.45, \mathrm{t}=$ -10.323 with $p<0.0001)$. Likewise, pain scores with cough were 3.29 units lower in the Block group than Non-block group (the estimate was $-3.29,95 \% \mathrm{Cl}=-3.80$ to $-2.77, \mathrm{t}=-12.703, \mathrm{p}<0.0001$ ). (Figure 3 ).

\section{Discussion}

Our study showed significantly decreased 24-hours fentanyl consumption with significantly prolonged time to first analgesic request in the block group than the non-block group. Also, we found reduced VAS scores of pain considerably both during rest and with cough after extubation in the block group than in the non-block group.

TTMP block can provide analgesia for post-sternotomy pain by targeting multiple anterior branches of the intercostal nerves (T 2-6). In their study, M.E. Aydin et al. ${ }^{(8)}$ reported a significant decrease in 24-hour fentanyl consumption among adult patients who received TTMP 
block compared to the control group. Also, various studies have reported the efficacy of TTMP block in reducing opioid consumption after cardiac surgery among the pediatric population. ${ }^{9}$, $10,11)$

On the other hand, Fujii et al. ${ }^{(12)}$ reported that the 24-hour opioid requirement was similar in patients who received or did not receive the block, this can be attributed to; first: their study was a pilot study and included only 19 patients which is a small number to declare a difference, second: lack of control of intraoperative and ICU opioid use which can affect postoperative pain scores and opioid requirement, furthermore, $60 \%$ of their patients underwent CABG with IMA harvesting. IMA harvesting will result in surgical disturbance of the TTMP block plane and uneven spread of the injectate between the desired thoracic levels. Patients may not benefit from the TTMP block on that side. ${ }^{(13)}$

We found that TTMP block significantly decreased VAS scores of pain at all-time intervals during the first 24 hours after extubation. Many studies have reported the same. $(9,10,11)$ However, M.E. Aydin et al. ${ }^{(8)}$ reported lower VAS scores only in the first 12 hours after extubation with no difference between block and non-block groups at 24 hours; this can be attributed to the time of performing the block as they performed TTMP block before surgery.

We found no difference in extubation time between both groups. On the other hand, studies in pediatrics found a shorter time for extubation among patients who received the block. $(9,10,11)$ Extubation is not dependent only on pain control; other factors such as hemodynamic stability and complete reversibility of NMB can affect the extubation time.

We found no difference in the incidence of nausea or vomiting between both groups; this can be attributed to ICU protocols as all patients receive prophylactic antiemetics regularly. Also, other studies reported the same. $(9,14)$

We did not report any block-related complications. Also, in their studies, M.E. Aydin et al. ${ }^{(14)}$ $\&$ Fujii et al. ${ }^{(12)}$ wrote no block-related complications. However, in a case series of 299 consecutive TTMP block cases, Ueshima $\mathrm{H}$ et al. ${ }^{(15)}$ said two patients with infection around the injection site.

In our study, we found no significant differences between both groups in LOS. Also, M.E. Aydin et al. ${ }^{(14)} \&$ M. Cakmak and O. Isik ${ }^{(11)}$ have found the same. On the other hand I.I. Abdelbaser and N.A. Mageed (9) \& Y. Zhang et al. ${ }^{(10)}$ showed a shorter LOS in the block groups compared with non-block groups.

\section{Limitations}

Median sternotomy is not the only source of pain following cardiac surgery; chest tubes and visceral pain are considered other essential sources. In this study, we targeted only post-sternotomy pain; this can explain why patients in the block group requested opioids. Another limitation is the dependence on NCA based on clinical data for supplementary analgesia before extubation which could affect opioid consumption. Furthermore, all participants were Egyptians, and CABG surgeries were not included due to anatomical considerations, limiting our data's generalizability. Again, the limited number of available clinical trials represented a difficulty for comparison. Despite these limitations, our results highlight the successful role of TTMP block in reducing pain and opioid consumption following median sternotomy.

\section{Conclusion}


TTMP block successfully reduces postoperative opioid consumption, prolongs time to first analgesic request, and decreases pain scores. It has an opioid-sparing effect and can be used as a part of a multimodal analgesia regimen in a patient undergoing open-heart surgeries via median sternotomy. We recommend further clinical trials over a large scale population to ensure the clinical role of TTMP block as apart from the multimodal analgesic regimen. Also, further studies are still requested to evaluate the possibility and efficacy of catheter placement for multiple injections.

\section{Abbreviations}

ASD $=$ Atrial septal defect

AVR=Aortic valve replacement

BMl=body mass index

$\mathrm{CPB}=$ Cardio-pulmonary bypass

DM=Diabetus Mellitus

$E F=$ ejection fraction

HTN= Hypertension

$\mathrm{ICU}=$ Intensive Care Unit

$\mathrm{IQR}=$ interquartile range

$M V R=$ Mitral valve replacement

PIF= Pecto intercostal fascial

$\mathrm{SD}=$ Standard deviation

TTMP = Transversus thoracic muscle plane

\section{Declarations}

\section{Ethics approval and consent to participate:}

The study design was approved by the ethical review board of Fayoum University Hospital (D 199), and written informed consent was acquired from all patients.

\section{Consent for publication:}

Written informed consent was attained from all patients 
Availability of data and materials:

The datasets used and/or analysed during the current study are available from the corresponding author on reasonable request.

\section{Competing interests:}

The authors declare no conflicts of interest.

\section{Funding:}

The authors have no sources of funding to declare for this manuscript.

\section{Authors' contributions}

Data collection: all authors

Data analysis: all authors

Writing: all authors

Revising: all authors

Study design: all authors

Patient recruitment: all authors

All authors contributed equally to this work.

Acknowledgements: Not applicable

\section{References}

1. Zubrzycki M, Liebold A, Skrabal C, Reinelt H, Ziegler M, Perdas E, Zubrzycka M. Assessment and pathophysiology of pain in cardiac surgery. J. Pain Res. [Internet]. Dove Press; 2018 Aug;Volume 11:1599-611. doi:10.2147/JPR.S162067.

2. Bignami E, Castella A, Pota V, Saglietti F, Scognamiglio A, Trumello C, Pace MC, Allegri M. Perioperative pain management in cardiac surgery: a systematic review. Minerva Anestesiol. [Internet]. 2018;84(4):488-503. doi:10.23736/S0375-9393.17.12142-5.

3. Kwanten LE, O'Brien B, Anwar S. Opioid-Based Anesthesia and Analgesia for Adult Cardiac Surgery: History and Narrative Review of the Literature. J. Cardiothorac. Vasc. Anesth. [Internet]. Elsevier; 2019 Mar;33(3):808-16. doi:10.1053/j.jvca.2018.05.053.

4. Caruso TJ, Lawrence K, Tsui BCH. Regional anesthesia for cardiac surgery. Curr. Opin. Anaesthesiol. [Internet]. Elsevier; 2019 Oct;32(5):674-82. doi:10.1097/AC0.0000000000000769. 
5. Ueshima H, Kitamura A. Blocking of Multiple Anterior Branches of Intercostal Nerves (Th2-6) Using a Transversus Thoracic Muscle Plane Block. Reg. Anesth. Pain Med. [Internet]. 2015;40(4):388. doi:10.1097/AAP.0000000000000245.

6. Ueshima $\mathrm{H}$, Takeda $\mathrm{Y}$, Ishikawa $\mathrm{S}$, Otake $\mathrm{H}$. Ultrasound-guided transversus thoracic muscle plane block: a cadaveric study of the spread of injectate. J. Clin. Anesth. [Internet]. Elsevier B.V.; 2015;27(8):696. doi:10.1016/j.jclinane.2015.05.013.

7. Byrne K, Cutts T. Fascial Plane Blocks: The Rise of the "Good- Enough" Block. J. Cardiothorac. Vasc. Anesth. [Internet]. Elsevier; 2020 Nov;34(11):3004-5. doi:10.1053/j.jvca.2020.07.068.

8. Aydin ME, Ahiskalioglu A, Ates I, Tor IH, Borulu F, Erguney OD, Celik M, Dogan N. Efficacy of Ultrasound-Guided Transversus Thoracic Muscle Plane Block on Postoperative Opioid Consumption After Cardiac Surgery: A Prospective, Randomized, Double-Blind Study. J Cardiothorac Vasc Anesth. 2020 Nov;34(11):2996-3003. doi: 10.1053/j.jvca.2020.06.044. Epub 2020 June 18. PMID: 32665179.

9. Abdelbaser II, Mageed NA. Analgesic efficacy of ultrasound guided bilateral transversus thoracis muscle plane block in pediatric cardiac surgery: a randomized, double-blind, controlled study. J. Clin. Anesth. [Internet]. Elsevier; 2020 Dec;67:110002. doi:10.1016/j.jclinane.2020.110002.

10. Zhang Y, Chen S, Gong H, Zhan B. Efficacy of Bilateral Transversus Thoracis Muscle Plane Block in Pediatric Patients Undergoing Open Cardiac Surgery. J. Cardiothorac. Vasc. Anesth. [Internet]. Elsevier; 2020 Sep;34(9):2430-4. doi:10.1053/j.jvca.2020.02.005.

11. Cakmak M, Isik O. Transversus Thoracic Muscle Plane Block for Analgesia After Pediatric Cardiac Surgery. J. Cardiothorac. Vasc. Anesth. [Internet]. Elsevier Inc.; 2020;000. doi:10.1053/j.jvca.2020.07.053.

12. Fujii S, Roche M, Jones PM, Vissa D, Bainbridge D, Zhou JR. Transversus thoracis muscle plane block in cardiac surgery: a pilot feasibility study. Reg. Anesth. Pain Med. [Internet]. BMJ Publishing Group Ltd; 2019 May;44(5):556-60. doi:10.1136/rapm-2018-100178.

13. Fujii S, Vissa D, Ganapathy S, Johnson M, Zhou J. Transversus Thoracic Muscle Plane Block on a Cadaver With History of Coronary Artery Bypass Grafting. Reg. Anesth. Pain Med. [Internet]. Lippincott Williams and Wilkins; 2017 July 1;42(4):535-7. doi:10.1097/AAP.0000000000000607.

14. Aydin ME, Ahiskalioglu A, Ates I, Tor IH, Borulu F, Erguney OD, Celik M, Dogan N. Efficacy of Ultrasound-Guided Transversus Thoracic Muscle Plane Block on Postoperative Opioid Consumption After Cardiac Surgery: A Prospective, Randomized, Double-Blind Study. J. Cardiothorac. Vasc. Anesth. [Internet]. Elsevier Inc.; 2020 Nov;34(11):2996-3003. doi:10.1053/j.jvca.2020.06.044.

15. Ueshima $\mathrm{H}$, Otake $\mathrm{H}$. Ultrasound-guided transversus thoracic muscle plane block: Complication in 299 consecutive cases. J. Clin. Anesth. [Internet]. Elsevier Inc.; 2017;41:60. doi:10.1016/j.jclinane.2017.03.056.

\section{Tables}

Table 1 Demographic Characteristics, Operative Data, and length of stay 


\begin{tabular}{|c|c|c|}
\hline & Group Block & Group No block \\
\hline Sample size „n & 35 & 35 \\
\hline Mean age (SD) in (years) & $38(15)$ & $39(13)$ \\
\hline Mean BMI (SD) in $\mathrm{kg} / \mathrm{m}^{2}$ & $25(4)$ & $25(3)$ \\
\hline \multicolumn{3}{|l|}{ Sex, n (\%) } \\
\hline Male & $22(63)$ & $22(63)$ \\
\hline Female & $13(37)$ & $13(37)$ \\
\hline \multicolumn{3}{|l|}{ HTN, n (\%) } \\
\hline Yes & $6(17)$ & $7(20)$ \\
\hline No & $29(83)$ & $28(80)$ \\
\hline \multicolumn{3}{|l|}{$\mathrm{DM}, \mathrm{n}(\%)$} \\
\hline Yes & $4(11)$ & $6(17)$ \\
\hline No & $31(89)$ & $29(83)$ \\
\hline \multicolumn{3}{|l|}{ The operation, $n(\%)$} \\
\hline ASD & $1(3)$ & $4(11)$ \\
\hline AVR & $7(20)$ & $10(29)$ \\
\hline MVR & $19(54)$ & $20(57)$ \\
\hline MVR \& AVR & $6(17)$ & $1(3)$ \\
\hline MVR \& ASD & $1(3)$ & $0(0)$ \\
\hline Triple valve & $1(3)$ & $0(0)$ \\
\hline Median Time of extubation (IQR) in hours & $5(4-6)$ & $6(5-6)$ \\
\hline Median ICU stay(IQR) in hours & $36(36-48)$ & $36(36-60)$ \\
\hline Median Hospital stay(IQR) in days & $4(3-5)$ & $4(3-4)$ \\
\hline
\end{tabular}

Abbreviations: SD, Standard deviation; n, number; ASD, Atrial septal defect; AVR, Aortic valve replacement; BMI, body mass index; D.M., Diabetes Mellitus; HTN, Hypertension; ICU, Intensive care unit; MVR, Mitral valve replacement; IQR, interquartile range.

Table 2 Total fentanyl consumption in the first 24 hours 


\begin{tabular}{|lllll|}
\hline & Block group & $\begin{array}{l}\text { Non-block } \\
\text { group }\end{array}$ & $\begin{array}{l}\text { Mean difference } \\
\mathbf{9 5 \%} \mathrm{Cl}\end{array}$ & $\begin{array}{l}\text { P-value } \\
\text {, }\end{array}$ \\
\hline Sample size , $\mathrm{n}$ & 35 & 35 & & \\
\hline $\begin{array}{l}\text { Mean total fentanyl } \\
\text { consumption (SD) in }(\boldsymbol{\mu})\end{array}$ & $205.7(73.5)$ & $390.9(80.3)$ & $\begin{array}{l}-185.143(-221.871 \text { to } \\
-148.415)\end{array}$ & $<0.0001^{*}$ \\
\hline
\end{tabular}

Abbreviations: SD, Standard deviation; n, number.

a: P-value compares block group versus the non-block group.

b: Two samples t-test used to compare means.

\section{Figures}

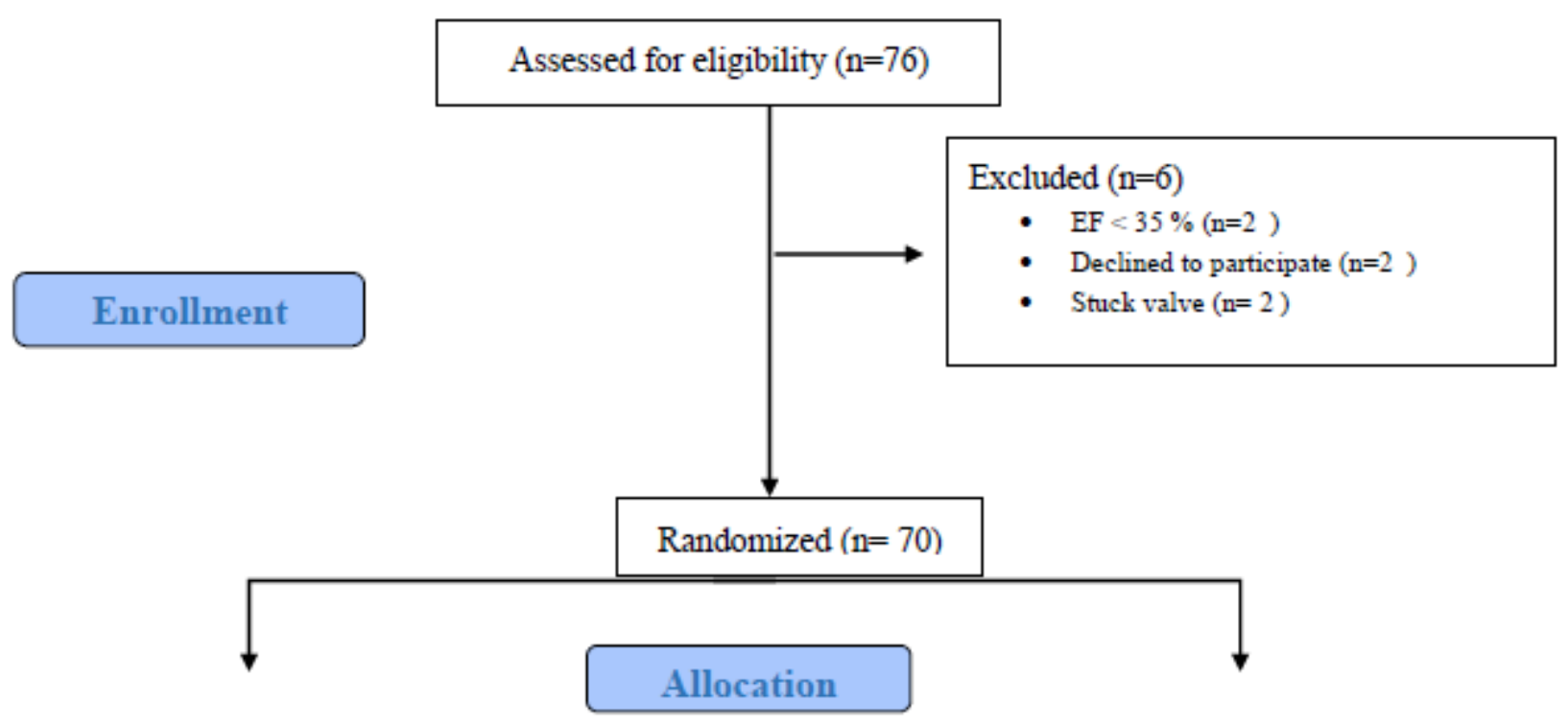

Randomly allocated to intervention group (block group) $(\mathrm{n}=35)$
Randomly allocated to control group

(Non-block group) $(\mathrm{n}=35)$

\section{Analysis}

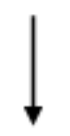

Analysed $(\mathrm{n}=35)$
Analysed ( $\mathrm{n}=35)$ 
Consort flow diagram of the study population.

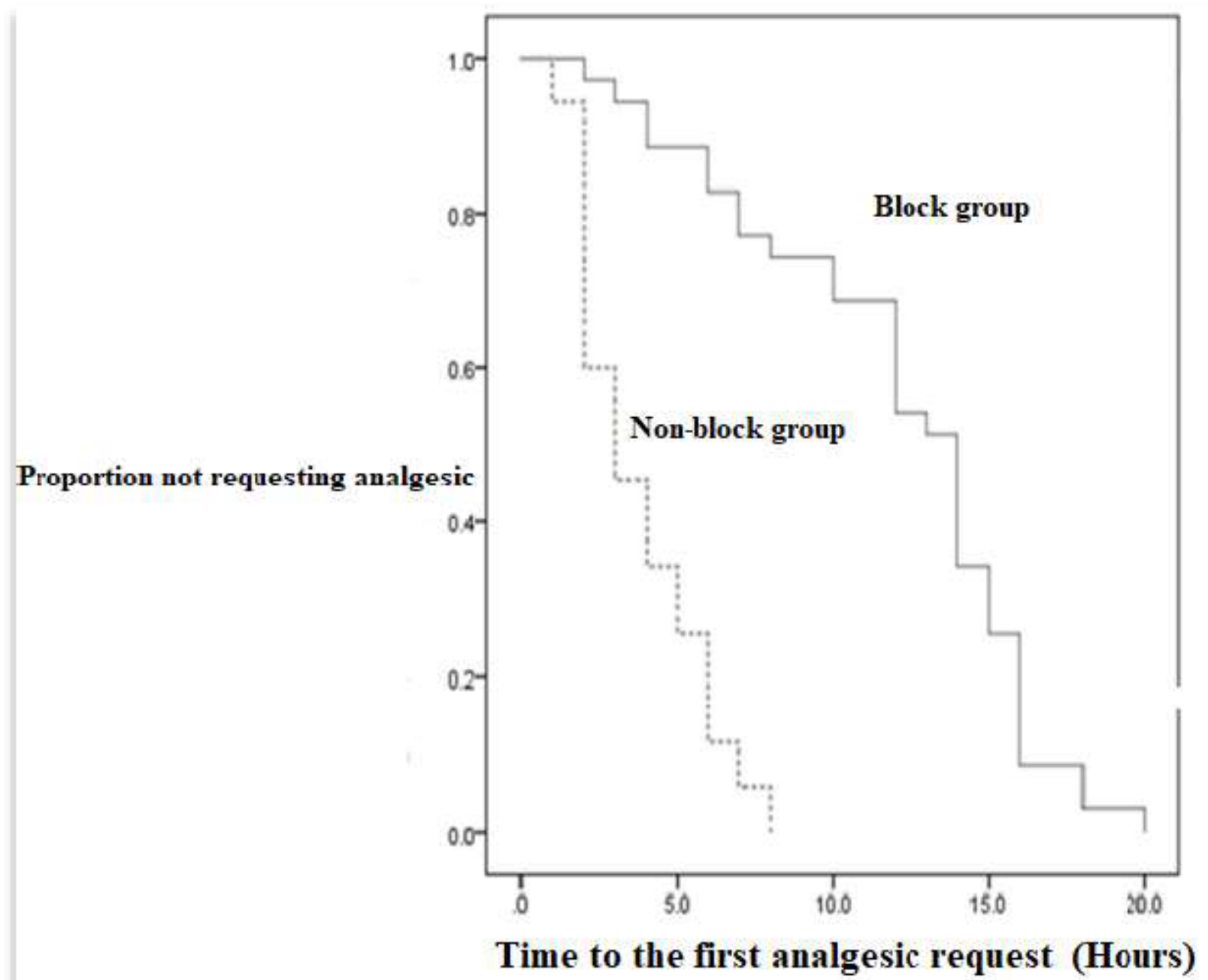

Figure 2

The time to the first analgesic request. 


\section{VAS score at rest}

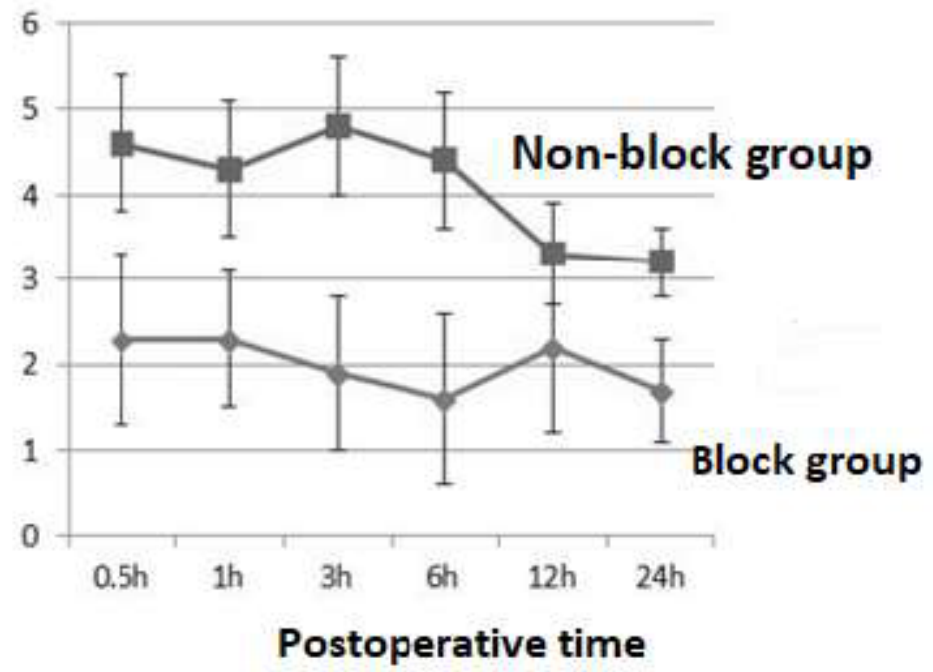

\section{VAS score with cough}

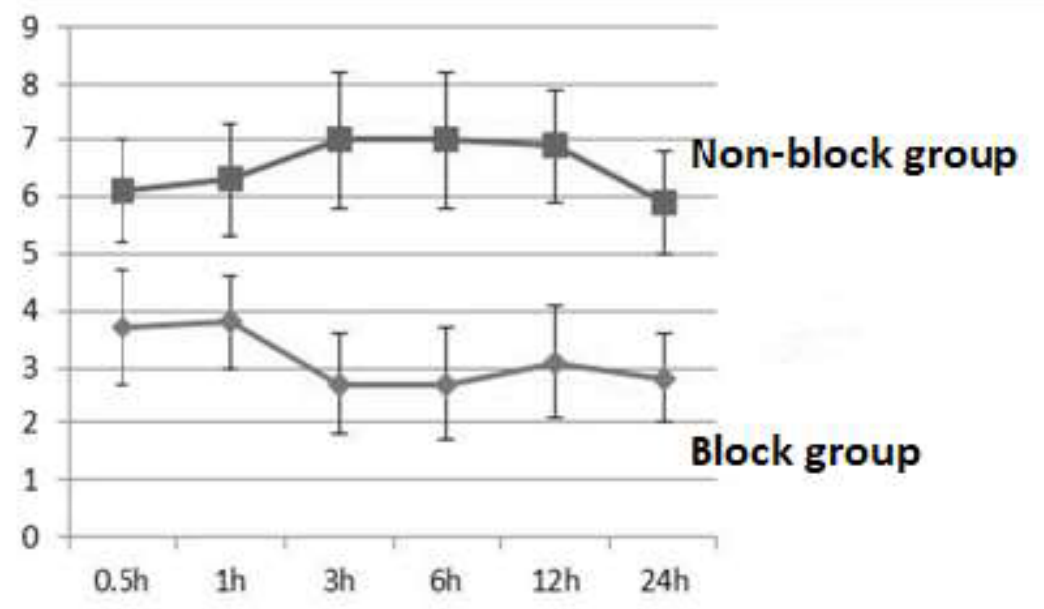

Postoperative time

Figure 3

The VAS score at rest and with cough. 